\title{
MOLECULAR DYNAMICS MODELING OF ATOMIC DISPLACEMENT CASCADES IN 3C- SiC: COMPARISON OF INTERATOMIC POTENTIALS
}

\author{
G. D. Samolyuk*, Y. N. Osetskiy and R. E. Stoller \\ Materials Science and Technology Division, Oak Ridge National Laboratory, USA \\ * Corresponding author: samolyukgd@ornl.gov (G.D. Samolyuk)
}

\begin{abstract}
We used molecular dynamics modeling of atomic displacement cascades to characterize the nature of primary radiation damage in $3 \mathrm{C}-\mathrm{SiC}$. We demonstrated that the most commonly used interatomic potentials are inconsistent with ab initio calculations of defect energetics. Both the Tersoff potential used in this work and a modified embedded-atom method potential reveal a barrier to recombination of the carbon interstitial and carbon vacancy which is much higher than the density functional theory (DFT) results. The barrier obtained with a newer potential by Gao and Weber is closer to the DFT result. This difference results in significant differences in the cascade production of point defects. We have completed both $10 \mathrm{keV}$ and $50 \mathrm{keV}$ cascade simulations in $3 \mathrm{C}-\mathrm{SiC}$ at a range of temperatures. In contrast to the Tersoff potential, the Gao-Weber potential produces almost twice as many $\mathrm{C}$ vacancies and interstitials at the time of maximum disorder $(\sim 0.2 \mathrm{ps})$ but only about $25 \%$ more stable defects at the end of the simulation. Only about $20 \%$ of the carbon defects produced with the Tersoff potential recombine during the in-cascade annealing phase, while about $60 \%$ recombine with the Gao-Weber potential. The Gao-Weber potential appears to give a more realistic description of cascade dynamics in $\mathrm{SiC}$, but still has some shortcomings when the defect migration barriers are compared to the ab initio results.
\end{abstract}

Research sponsored by the Office of Fusion Energy Sciences, U.S. Department of Energy, under contract DE-AC05-00OR22725 with UT-Battelle, LLC. Accordingly, the U.S. Government retains a nonexclusive, royalty-free license to publish or reproduce the published form of this contribution, or allow others to do so, for U.S. Government purposes. 


\section{INTRODUCTION}

The outstanding physical, chemical and mechanical properties of silicon carbide (SiC) have stimulated significant technological and scientific interest for its use in electronic devices and neutron irradiation environments [1]. When subjected to high energy neutron irradiation, the microstructure and hence the mechanical properties of 3C-SiC (cubic phase of SiC) are changed. Understanding the details of this process is necessary for predicting these changes, and estimating the material's usable lifetime. Experimental studies have demonstrated that depending on the structural material's composition and other particular conditions, phenomena such as amorphization [2-7] and swelling [8-10] are observed.

Currently, the molecular dynamics approach with the atom-atom interactions described by empirical interatomic potentials appears to be the main tool for simulating atomic displacement cascades. In this approach, the results of cascade modeling significantly depends upon the accuracy of the description of the main defect properties by the interatomic potential. In the present paper we give a detailed comparison of the results of atomic displacement cascades in $\mathrm{SiC}$ obtained using the popular Tersoff [11] potential, with the parametrization proposed by Devanathan et al. [12] with the more recent GaoWeber (GW) potential [13]. Despite having reasonably good agreement with the native defect formation energies obtained from ab initio density functional theory (DFT) calculations [14], the Tersoff potential predicts a very high interstitial migration energy [13]. We demonstrate that such high barrier also significantly suppressed the recombination of the carbon interstitial-vacancy defect pair, even when separated by distances as small as one lattice parameter. It is shown that this difference in barrier recombination also leads to a different temperature dependence for the number of stable defects at the end of cascade evolution.

\section{SIMULATION METHOD}

We used the LAMMPS code (Large-scale Atomic/Molecular Massively Parallel Simulator; see http://lammps.sandia.gov) for atomistic molecular dynamics simulations [15]. The interactions between atoms were described by either the Tersoff [12] or GW [13] empirical potentials. In order to improve the weak repulsion of these potentials at short distances, they are typically joined with the well-established Ziegler, Biersack and Littmark (ZBL) potential [16] at $0.095 \mathrm{~nm}$ using a Fermi-like function, as realized in LAMMPS, see Figure 1. 
Simulations involving 80x80x80 unit cells (409,600 atoms) were used for $10 \mathrm{keV}$ primary cascades and 150x150x150 unit cells $(22,070,021$ atoms) for $50 \mathrm{keV}$ cascades. The initial system was equilibrated for 2 picoseconds with time steps of 0.1 femtoseconds. Each cascade was initiated by giving a Si atom the kinetic energy of 10 or $50 \mathrm{keV}$ in a direction close to $\langle 135\rangle$ while holding zero total momentum. The cascades evolved for 20 ps and the time step is modified such that the distance traveled by the fastest particle in the system is less than $0.0014 \mathrm{~nm}$ per timestep. Constant volume is maintained throughout the simulation and the lattice parameter is chosen maintain zero system pressure in equilibrium at a particular temperature. The Wigner-Seitz cell analysis method was used to determine defects in the modeling system.

The $a b$ initio calculations of the recombination barrier were carried out using DFT and the Quantum ESPRESSO (QE) package [17]. The calculations have been done using a plane-wave basis set and an ultra-soft pseudo-potential [18] optimized in the Rappe-Rabe-Kaxiras-Joannopoulos (RRKJ) scheme [19] (Si.pbe-n-rrkjus.UPF, C.pbe-n-rrkjus.UPF from the QE pseudopotentials database). We used the exchange-correlation functional parametrization according to Perdew, Burke, and Ernzerhof [20]. The Brillouin zone summations were carried out over a 4x4x4 Brillouin zone grid for the system with 64 atoms in the supercell. Electronic smearing with a width of 0.02 Ry was applied according to the Methfessel-Paxton method. Using a plane wave energy cut-off of 40 Ry and charge density of 400 Ry allows reaching an accuracy of $0.2 \mathrm{mRy} / \mathrm{atom}$. Atomic structure was optimized until the forces were smaller than $0.001 \mathrm{Ry} / \AA$.

\section{RESULTS AND DISCUSSION}

The $10 \mathrm{keV}$ cascade simulations with the Tersoff potential were carried out for a range of temperatures and indicated that in-cascade recombination in $\mathrm{SiC}$ seemed to be much lower than in metals. In Figure 2, we show the number of defects as a function of time in $50 \mathrm{keV}$ cascade in SiC (Figure 2a) and 10, 20 and $40 \mathrm{keV}$ in Fe (Figure 2b). The ratio of the peak defect count to the stable defect count at $\mathrm{t}>10$ picoseconds is less than a factor of two in $\mathrm{SiC}$ and almost two orders of magnitude in iron. Such a significant difference between $\mathrm{SiC}$ and $\mathrm{Fe}$ is due to specific properties of the $\mathrm{Si}-\mathrm{C}$ interaction. In $\mathrm{SiC}$, a strong-bond oriented interaction allows creation of a stable structure if each atom has four nearest neighbors. The second neighbors are placed at distances, which are $60 \%$ greater when compared to the nearest neighbors. As a result, the amorphous phase is very close in energy to the crystalline one and it's 
very easy to create metastable interstitial-vacancy pairs with a sizable recombination barrier. In the case of Si these interstitial and vacancy pairs produce a so-called I-V complex [21-24].

Point defects in $\mathrm{SiC}$ consist of interstitials (denoted as $\mathrm{C}(\mathrm{I})$ and $\mathrm{Si}(\mathrm{I})$ ) and vacancies $(\mathrm{C}(\mathrm{V})$ and $\mathrm{Si}(\mathrm{V})$ ) of both carbon and silicon, as well as anti-site defects of $\mathrm{C}$ on a $\mathrm{Si}$ site $\left(\mathrm{C}_{\mathrm{Si}}\right)$ and $\mathrm{Si}$ on a $\mathrm{C}$ site $\left(\mathrm{Si}_{\mathrm{C}}\right)$. Similar to previous results $[25,26]$, it was observed that carbon defects predominate for both the Tersoff and $\mathrm{GW}$ potentials, as shown by the green line with up triangles $(\mathrm{C}(\mathrm{I}))$ and purple line with open circles (C(V)) symbols in Figure 3a,b for $10 \mathrm{keV}$ cascades at temperature $300 \mathrm{~K}$. The GW result (Figure $3 \mathrm{~b}$ ) is qualitatively similar to that obtained with the Tersoff potential (Figure 3a). However, in contrast to the simulations with the Tersoff potential, the simulations with the GW potential produces almost twice as many $\mathrm{C}$ vacancies and interstitials at the time of maximum disorder ( $\sim 0.2 \mathrm{ps})$, but has only about $25 \%$ more stable defects at the end of the simulation. As a result, the ratio of peak-to-stable defects is much higher for the GW. This result is more similar to that observed in metals. Only about $20 \%$ of the carbon defects produced with the Tersoff potential recombine during the in-cascade annealing phase, while about $50 \%$ recombine with the GW potential. To emphasize the differences, the results for silicon (circles) and carbon (triangles) vacancies only are presented in Figure 3c. The results obtained using Tersoff potential are shown by a blue dashed line and the GW with a solid red line.

In order to investigate the source of the stability of carbon atom I-V pairs in modeling using the Tersoff potential, we calculated the barrier to $\mathrm{C}$ atom migration from its initial position $(1 / 4,1 / 4,1 / 4)$ to a metastable position $(3 / 4,3 / 4,3 / 4)$ along the [111] direction shown in (Figure 4a). The resulting configuration corresponds to a $\mathrm{C}(\mathrm{I})-\mathrm{C}(\mathrm{V})$ distance between the I-V pair of $\sqrt{3} / 2 a_{\mathrm{o}}$. The DFT results are close to that presented in references $[27,28]$. The recombination barrier, which corresponds to $\mathrm{C}$ atom migration from the $(3 / 4,3 / 4,3 / 4)$ position to its initial $(1 / 4,1 / 4,1 / 4)$ position obtained has a very large value $(\sim 10 \mathrm{eV})$ for both Tersoff (shown green in Figure $4 \mathrm{~b})$ and another popular modified embedded atom method (MEAM; red color) potential [29], whereas the first principles calculations give a value equal to $0.2 \mathrm{eV}$ (shown in purple). This large barrier suppresses any migration in the modeling system. The GW potential more reasonably describes interstitial diffusion in $\mathrm{SiC}$; however, the migration barrier calculated with this potential (blue line in Figure 4b) is much less that the first principles result. During translation in the [111] direction, the carbon atom doesn't come closer than $0.1767 \mathrm{~nm}$ to either a silicon or another carbon atom. At this distance, the atomic interactions involve only the initial GW or Tersoff potential functions, i.e. this is beyond the range where the ZBL pair potential has any influence. 
A significant difference is also found between the cluster size distributions obtained with the two potentials. The cluster size distributions obtained from $10 \mathrm{keV}$ cascade simulations for both potentials are presented in Figure 5a and b. In our analysis we defined a group of point defects as a single linked cluster if all of the defects are within one lattice parameter of another defect. Simulations with either potential produce a significant number of pair clusters with $\sim 75 \%$ of these clusters involving an interstitial-vacancy pair of the same type of atom. However, in the case of the Tersoff potential, all I-V pair clusters involve carbon atoms, while with the GW potential a significant fraction of these clusters involve silicon atoms. Another difference observed is in the creation of large-size clusters. With the Tersoff potential (Figure 5a), there are only two relatively large clusters containing 19 and 21 defects. In contrast, simulations with the GW potential produced four large clusters containing 21, 30, 35 and 51 defects. These large clusters are traditionally considered to be potential candidates for amorphization domains [26]. The difference in the size distributions is illustrated in Figure 5c, where the defect cluster sizes account for all defect types in the cluster. Following the approach used for tungsten in Ref. [30], the clusters were grouped into bins with a constant logarithmic width, i.e. the ratio of the upper bin limit to the lower bin limit equals $\ln (2)$. The frequency-size distribution of the average number of clusters containing three or more point defects follows a power law as shown in Figure 5c and given by the equation:

$$
F(n)=\frac{1}{n^{s}}
$$

where $A=53.7 \pm 6.9$ and $S=1.1 \pm 0.1$ for the Tersoff, and $A=80.0 \pm 12.1$ and $S=1.2 \pm 0.1$ for the GW potential. The authors of Ref. [30] associate this power law with the fractal nature of cascade development. It should be mentioned that clustering in $\mathrm{SiC}$ is much more complicated than for bcc tungsten [30] because $\mathrm{SiC}$ has two chemically distinct types of interstitial atoms and vacancies, plus the antisite defects. The largest cluster from the GW simulations containing 51 defects is presented in Figure 6. The carbon defects predominate as shown by the green (C) and purple ( $\mathrm{Si}$ ) symbols

In Figure 7, we show the number of defects and mean-square displacements (MSD) of Si and C atoms as a function of time obtained with the GW potential in a $10 \mathrm{keV}$ cascade at an elevated temperature (1200 $\mathrm{K})$. After about 15 picoseconds, the number of remaining defects becomes approximately constant., At longer times, some further reduction in the number of vacancies and interstitials occurs and is accompanied by an increase in atomic mixing as shown by the number of antisite defects $\left(\mathrm{Si}_{\mathrm{Si}}\right.$ and $\mathrm{C}_{\mathrm{C}}$ 
defects in Figure 7) and a significant rise in MSD values. This stage of cascade evolution corresponds to in-cascade annealing and is normally not explored in MD calculations. It is can be observed at relatively short times here because of the high temperature. The Tersoff potential results don't demonstrate any annealing and the number of defects remain constant value after the initial saturation. In order to compare the number of stable defects at the end of cascade evolution between the two potentials, we have taken the $\mathrm{GW}$ values at 20 picoseconds.

The number of stable defects observed at the end of cascade evolution has been calculated as an average value from 8 cascades. The number for $10 \mathrm{keV}$ cascades is weakly temperature dependent when using the Tersoff potential (Figure 8a), and increases slightly with temperatures. In contrast, for the results from the GW potential (Figure 8b) have much stronger temperature dependence and decrease with temperature. The temperature dependence with GW is more similar to that observed in metals, although it is stronger [31]. The difference between the Tersoff and GW potentials is due to the artificially large migration barriers obtained from the Tersoff potential. An increase in temperature naturally leads to more diffusion of carbon vacancies and interstitials. As a result, more carbon vacancies and interstitials recombine and the number of defects at the end of cascade evolution is reduced as the temperature increases. In contrast, the defect migration barriers using the Tersoff potential are so large that diffusion is suppressed, even at temperatures as high as $1600 \mathrm{~K}$, and the number of defects is only weakly temperature dependent. The number of defects obtained in $50 \mathrm{keV}$ cascades calculated with both the GW and Tersoff potentials are shown in Figure 9, and exhibits a similar temperature dependence to the $10 \mathrm{keV}$ cascades.

\section{CONCLUSIONS}

We demonstrated that two of the most commonly used interatomic potentials for SiC (Tersoff and MEAM) are inconsistent with ab initio calculations of defect energetics. This results in a significant difference in the cascade production and stability of points defects. To compare the Tersoff [12] and Gao-Weber [13] potentials, we have completed both $10 \mathrm{keV}$ and $50 \mathrm{keV}$ cascade simulations in 3C-SiC at temperatures of $300,600,900$ and $1200 \mathrm{~K}$ in $3 \mathrm{C}-\mathrm{SiC}$. The primary results can be summarized as follows:

- The ratio of peak-to-stable defects is much higher for the GW potential than for the Tersoff potential; 
- The primary reason for the difference is that the Tersoff potential predicts a barrier to carbon atom vacancy-interstitial recombination which is much higher than DFT results, while the GW potential barrier is much closer to DFT.

- The temperature dependence of the number of stable defects produced (Tersoff: weak and increasing with temperature; GW: stronger and decreasing with temperature) is also caused by the recombination barrier difference.

- The GW potential appears to give a more realistic description of cascade dynamics in $\mathrm{SiC}$, but still has some shortcomings when the defect migration barriers are compared to the ab initio results.

\section{ACKNOWLEDGMENTS}

We are grateful to Dr. L. K. Béland for useful discussions. This work was supported by the US Department of Energy Office of Fusion Energy Sciences. Additional computational resources have been used for this work through collaboration with the Japan Atomic Energy Agency.

\section{REFERENCES}

1. M.A. Capano, R.J. Trew, MRS Bull. 22 (1997) 19.

2. H. Inui, H. Mori, and H. Fujita,. Philos. Mag. B 61 (1990) 107.

3. H. Inui, H. Mori, T. Sakata, and H. Fujita, Philos. Mag. B 65 (1992) 1.

4. E. Wendler, A. Heft, and W. Wesch, Methods Phys. Res., Sect. B 141 (1998) 105.

5. Y. Zhang, F. Gao, W. Jiang, D.E. McCready, and W.J. Weber, Phys. Rev. B 70 (2004) 125203.

6. W. Jiang, H. Wang, I. Kim, I-T. Bae, G. Li, P. Nachimuthu, Z. Zhu, Y. Zhang, and W.J. Weber, Phys. Rev. B 80 (2009) 161301.

7. L.L. Snead and J.C. Hay, J. Nucl. Mater. 273 (1999) 213.

8. R. Blackstone and E.H. Voice, J. Nucl. Mater. 39 (1971).

9. J.E. Palentine and J. Nucl. Mater. 61 (1976) 243.

10. A.I. Ryazanov, A.V. Klaptsov, A. Kohyama, H. Kishimoto, J. Nucl. Mater. 307-311 (2002) 1107.

11. J. Tersoff, Phys. Rev. B 39 (1989) 5566.

12. R. Devanathan, T. Diaz de la Rubia, W.J. Weber, J. Nucl. Mater. 253 (1998) 47.

13. F. Gao and W. J. Weber, Nucl. Instr. And Methods in Phys. Research B 191 (2002) 504. 
14. F. Gao, E. J. Bylaska, W. J. Weber, and L. R. Corrales, Nucl. Instr. and Methods in Phys. Research B 180 (2001) 286.

15. S. Plimpton, J. Comp. Phys. 117 (1995) 1.

16. J.F. Ziegler, J.P. Biersack, U. Littmark, The Stopping and Ranges of Ions in Solids. vol. 1, Pergamon, 1985.

17. P. Giannozzi, S. Baroni, and N. Bonini, J. of Physics: Condens. Mat. 21 (2009) 395502.

18. D. Vanderbilt, Phys. Rev. B 41 (1990) 7892.

19. A.M. Rappe, K.M. Rabe, E. Kaxiras, and J.D. Joannopoulos, Phys. Rev. B 41 (1990) 1227.

20. J.P. Perdew, K. Burke, and M. Ernzerhof, Phys. Rev. Lett. 77 (1996) 3865.

21. M. Tang, L. Colombo, J. Zhu, and T. Diaz de la Rubia, Phys. Rev. B 55 (1997) 14279.

22. F. Wooten, K. Winer, D. Weaire, Phys. Rev. Lett. 54 (1985) 1392.

23. L.K. Béland, and N. Mousseau, Phys. Rev. B 88 (2013) 214201.

24. L.K. Béland, Y. Anahory, D. Smeets, M. Guihard, P. Brommer, J.-F. Joly, J.-C. Pothier, L.J. Lewis, N. Mousseau, and F. Schiettekatte. Phys. Rev. Lett. 111(2013) 105502.

25. D.E. Farrell, N. Bernstein, and W.K. Liu, J. Nucl. Mater. 385 (2009) 572.

26. W.J. Weber, and F. Gao, J. Mater. Res., 25 (2010) 2349.

27. G. Lucas, and L Pizzagalli, J. Phys.: Cond. Matter. 19 (2007) 86208.

28. M.-J. Zheng, N. Swaminathan, D. Morgan, and I. Szlufarska, Phys. Rev. B 88 (2013) 54105.

29. H. Huang, N.M. Ghoniem, J.K. Wong, M.I. Baskes, Modeling Simul. Mater. Sci. Eng. 3 (1995) 615.

30. A.E. Sand, S.L. Dudarev, and K. Nordlund, Euro. Phys. Lett. 103 (2013) 46003.

31. R. E. Stoller, "Primary Radiation Damage Formation," in Comprehensive Nuclear Materials, R. J. M. Konings, T. R. Allen, R. E. Stoller, and S. Yamanaka, Eds., Elsevier Ltd., Amsterdam, 2012, pp. 293-332. 


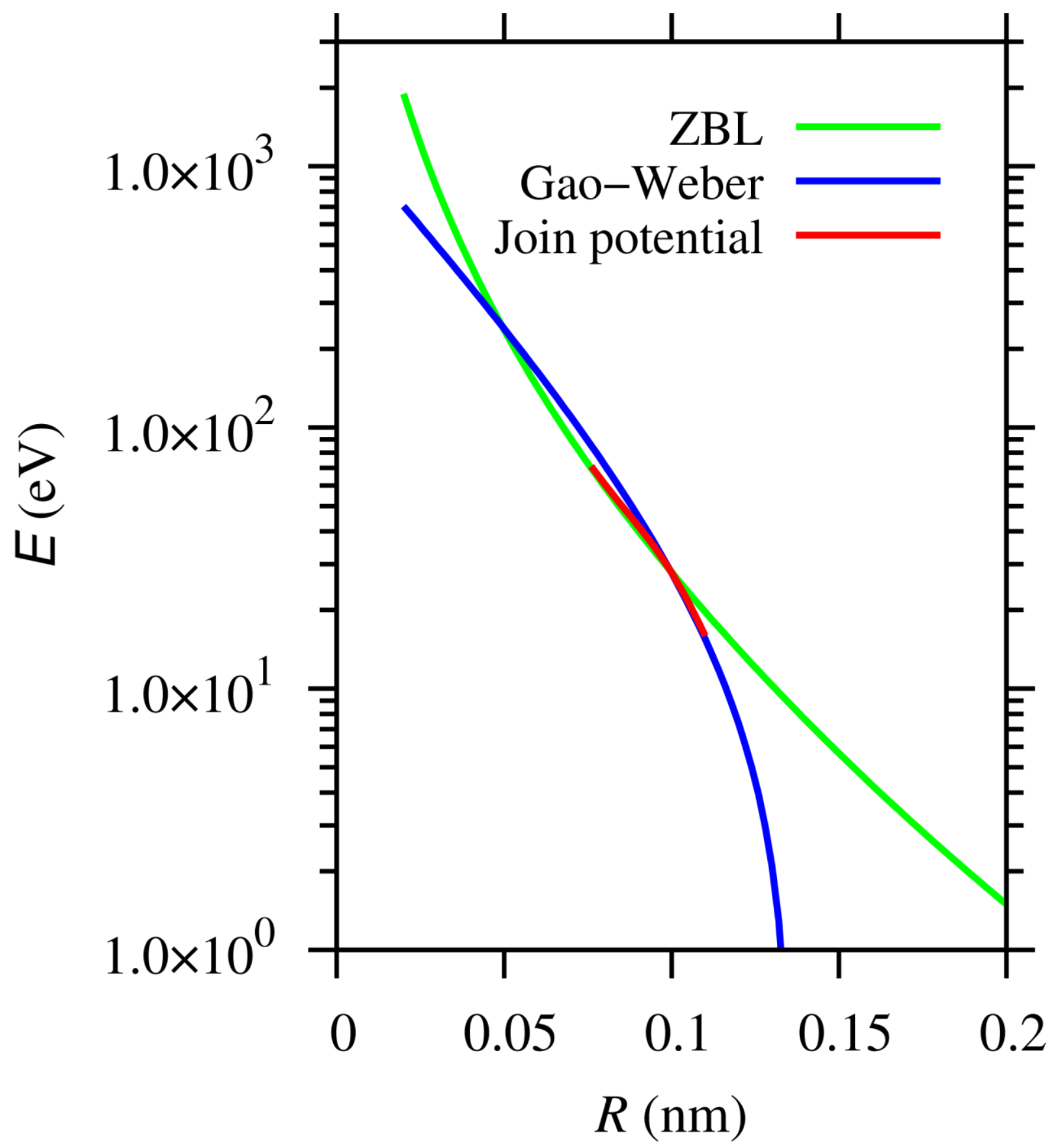

Figure 1. Repulsive Si-C atoms interaction. The GW potential is shown by blue color, the ZBL by green color and the resulting join potential is shown by red color line 
a)
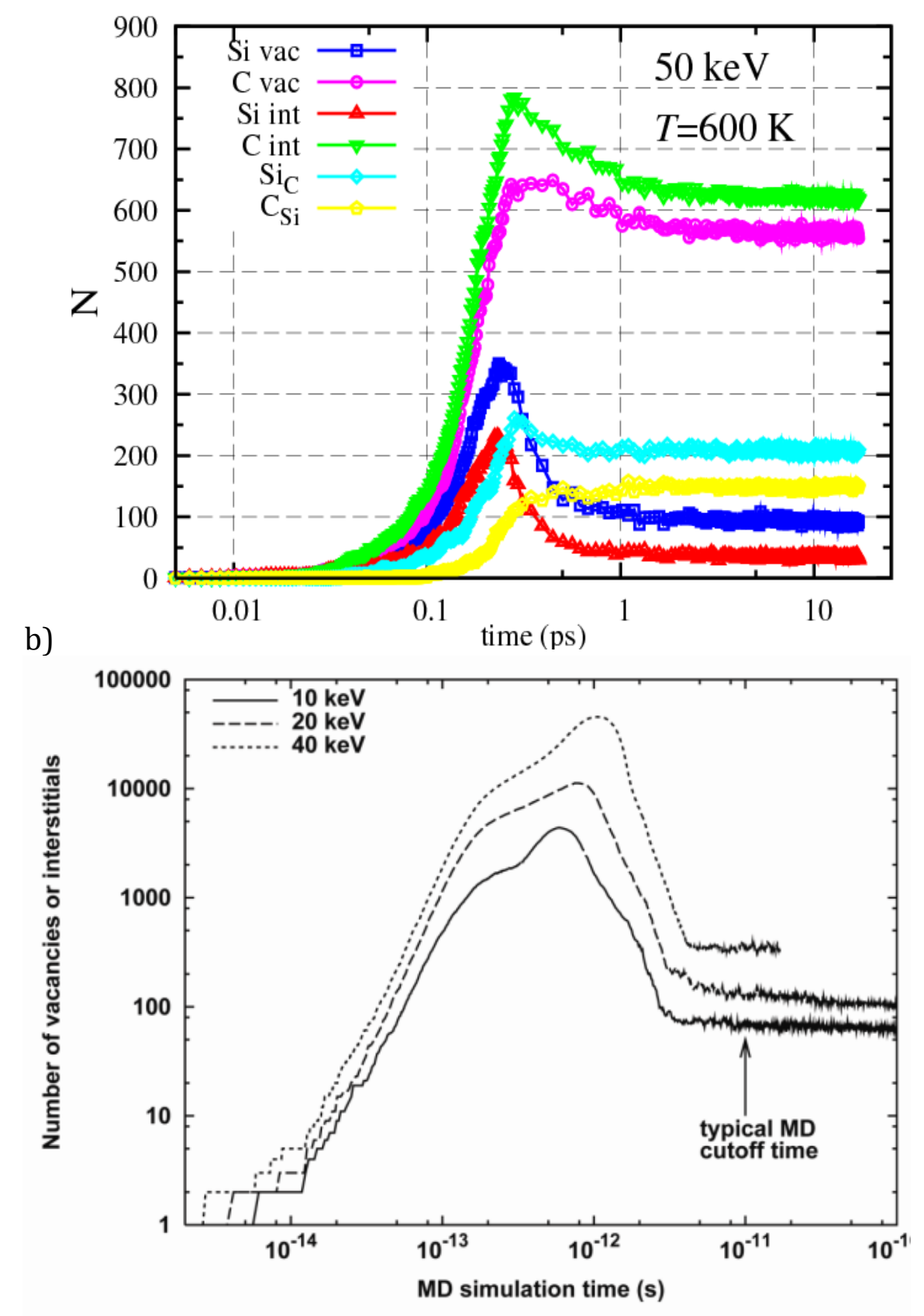

Figure 2. Time dependence of the number of point defects observed in MD displacement cascade simulations: (a) $50 \mathrm{keV} \mathrm{SiC}$ cascade at $600 \mathrm{~K}$ with Tersoff potential and (b) 10, 20, and $40 \mathrm{keV}$ cascades in iron 
a)

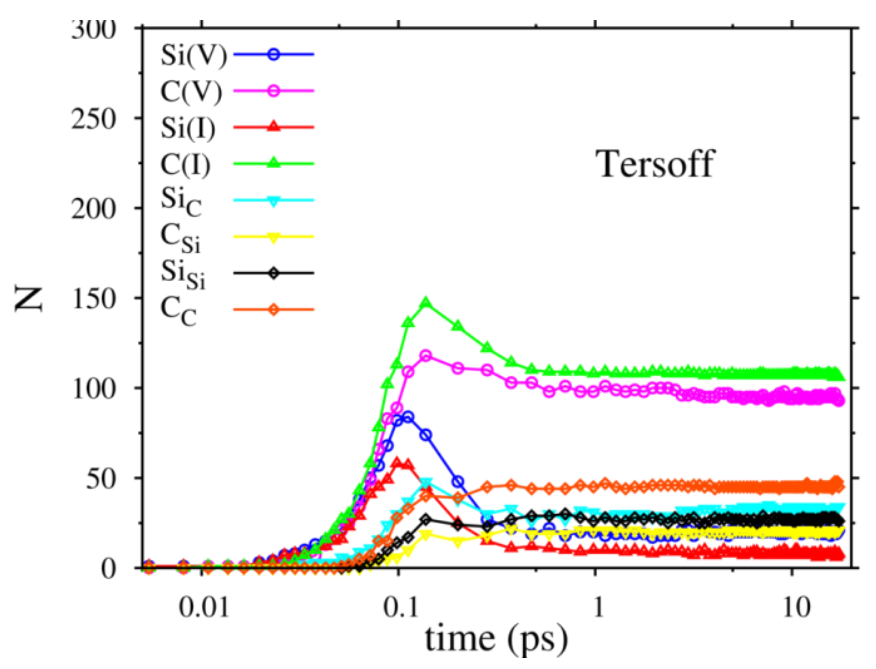

b)

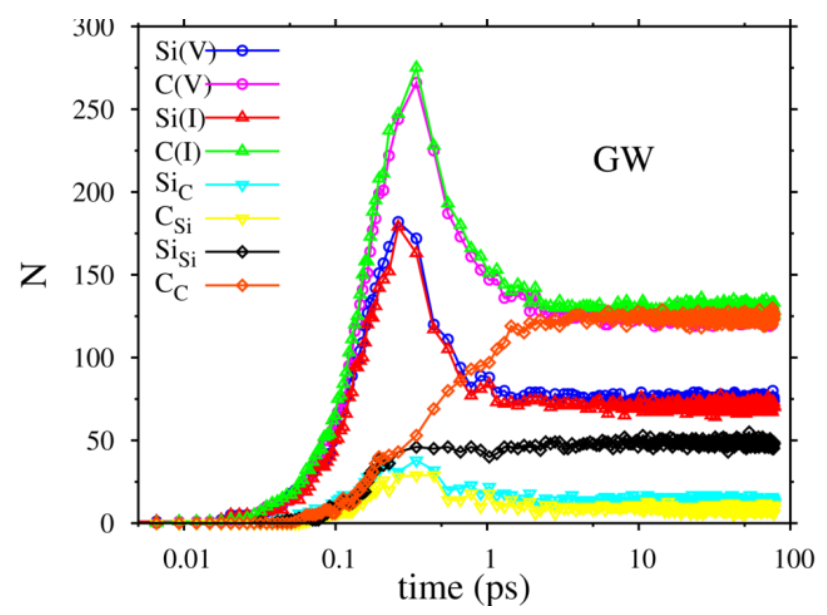

c)

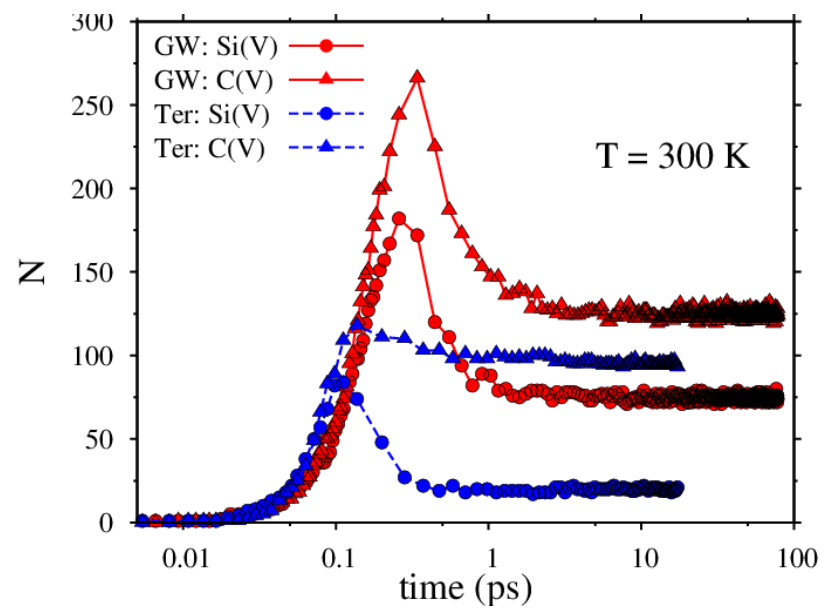

Figure 3. Time dependence of the number of point defects observed in MD displacement cascade simulations at $10 \mathrm{keV}$ pka at $300 \mathrm{~K}$ : (a) Tersoff potential, (b) GW potential, (c) both potentials showing results for vacancies only on the same plot. 
a)

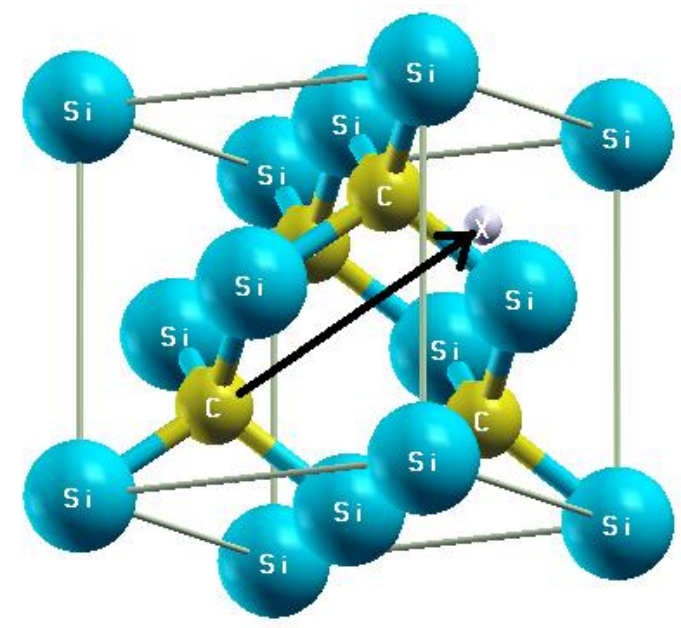

b)

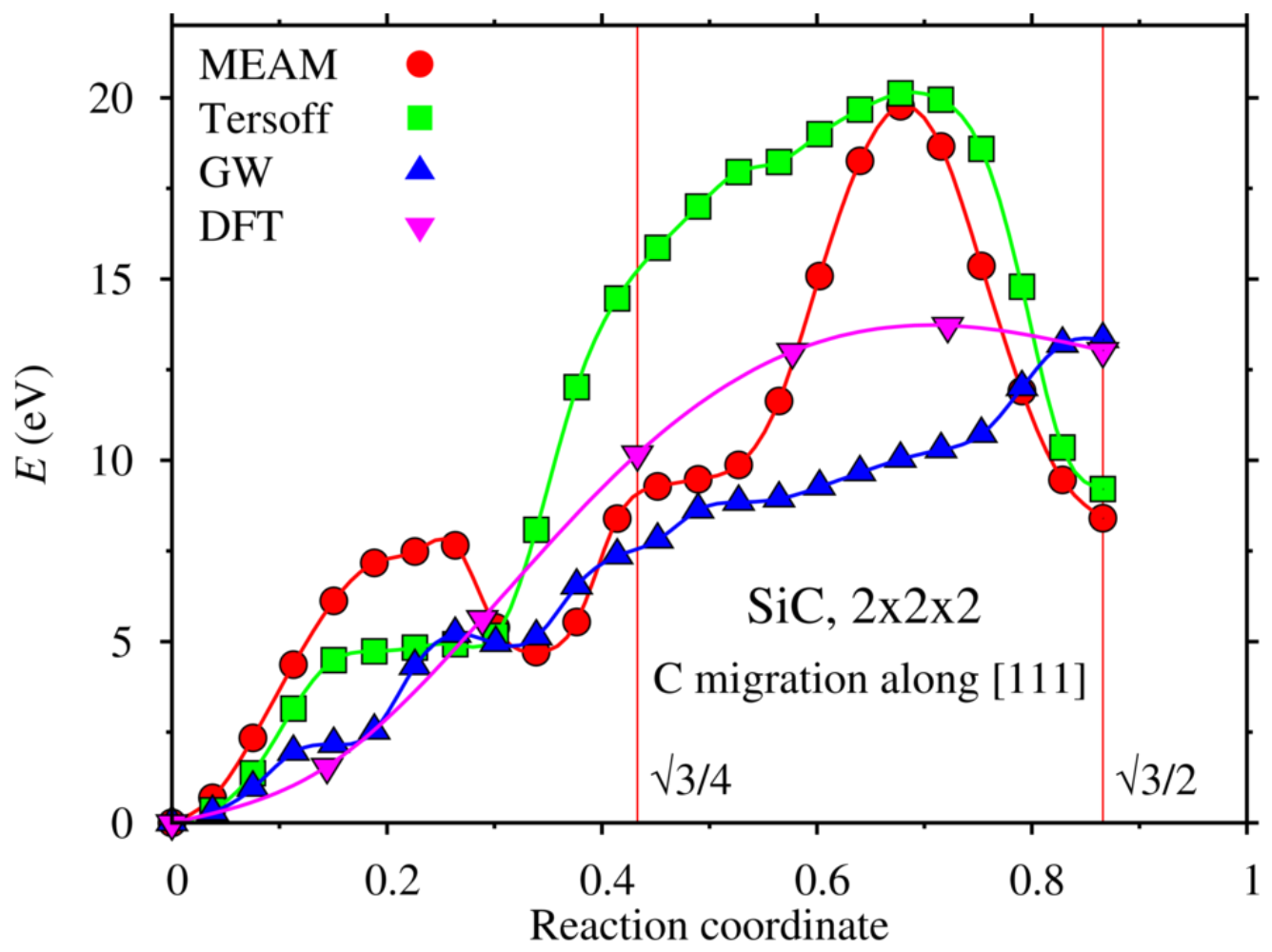

Figure 4. (a) Carbon atom migration path along [111] direction to the $\langle 111\rangle$ dumbbell configuration, (b) corresponding migration barrier 
a)

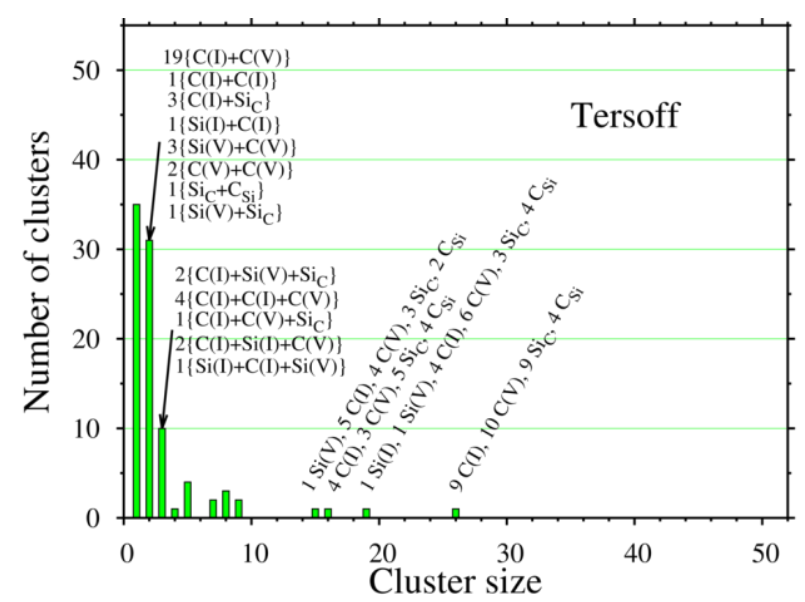

b)

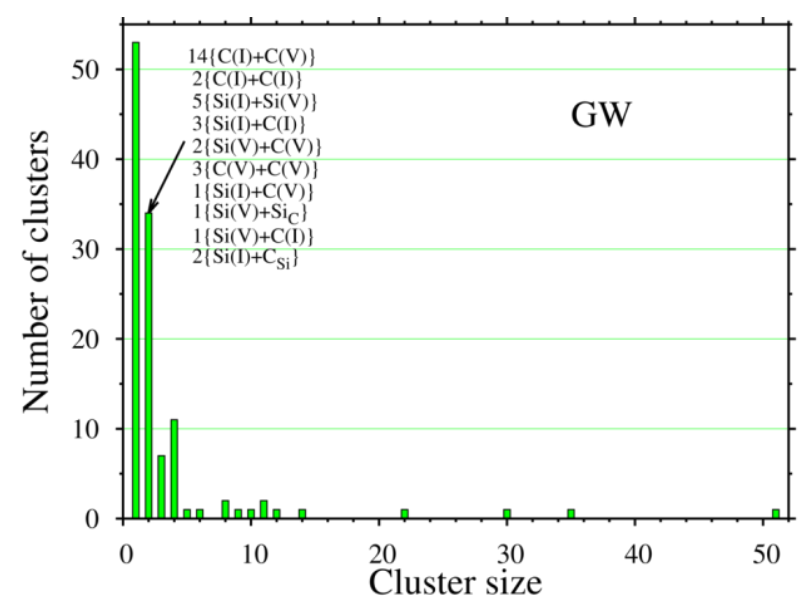

c)

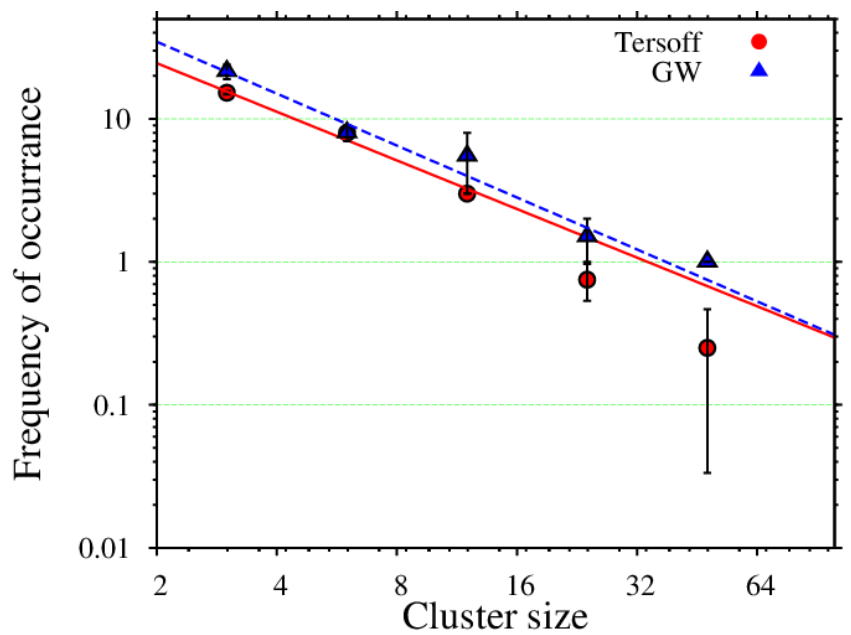

Figure 5. Cluster size distribution at the end of $10 \mathrm{keV} \mathrm{Si} \mathrm{recoil} \mathrm{event} \mathrm{in} \mathrm{SiC} \mathrm{at} 300 \mathrm{~K}$ : (a) Tersoff potential, (b) GW potential, (c) frequency-size distribution of clusters with three or more point defects. The data were averaged using bins with equal logarithmic widths and fitted to a power function. 


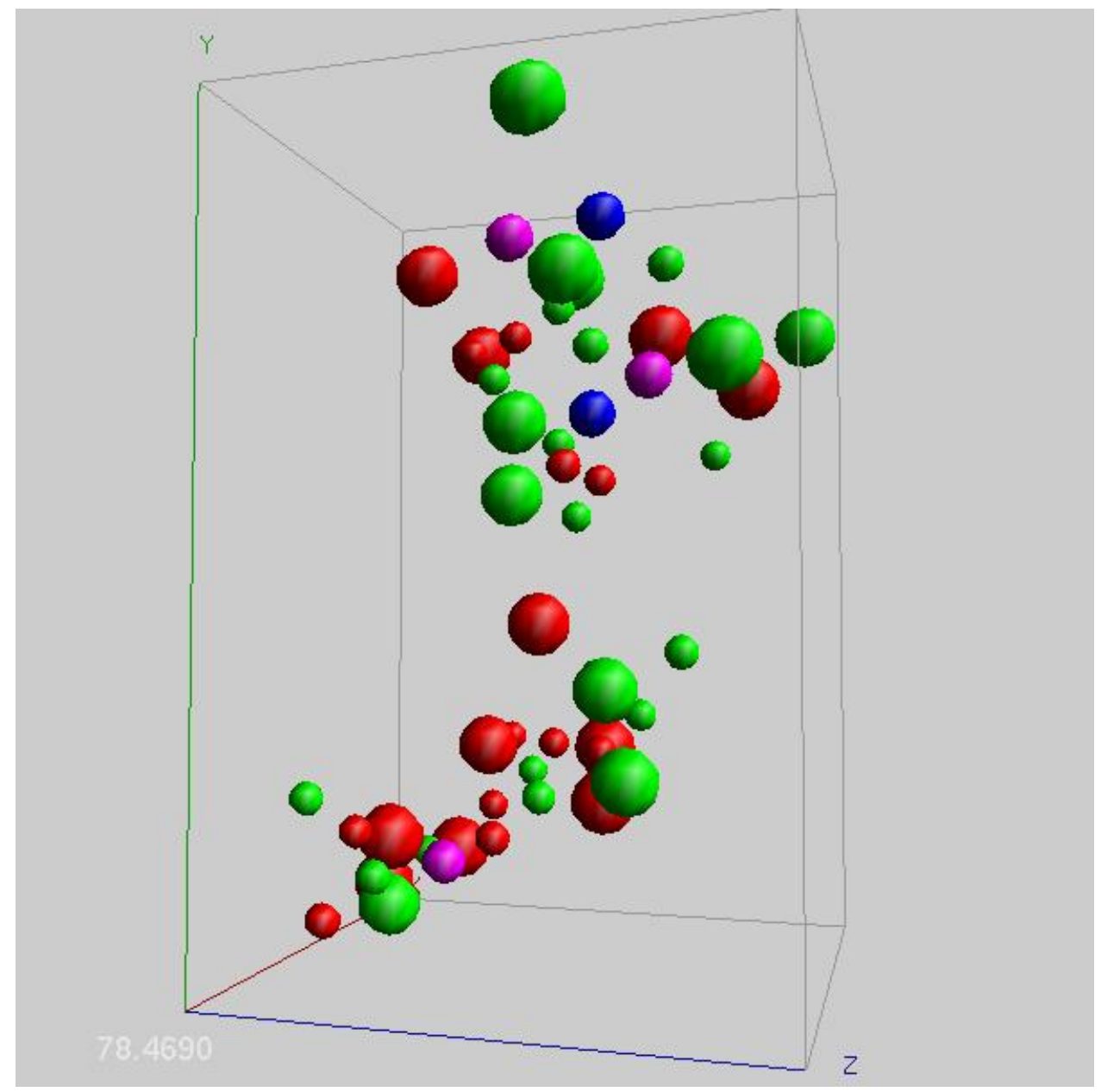

Figure 6. Carbon (green) and silicon (red) interstitials (large spheres) and vacancies (small spheres) obtained from a $10 \mathrm{keV}$ Si recoil event in $\mathrm{SiC}$ with the $\mathrm{GW}$ potential. Blue and purple spheres correspond to $\mathrm{Si}_{\mathrm{C}}$ and $\mathrm{C}_{\mathrm{Si}}$ anti-sites, respectively 


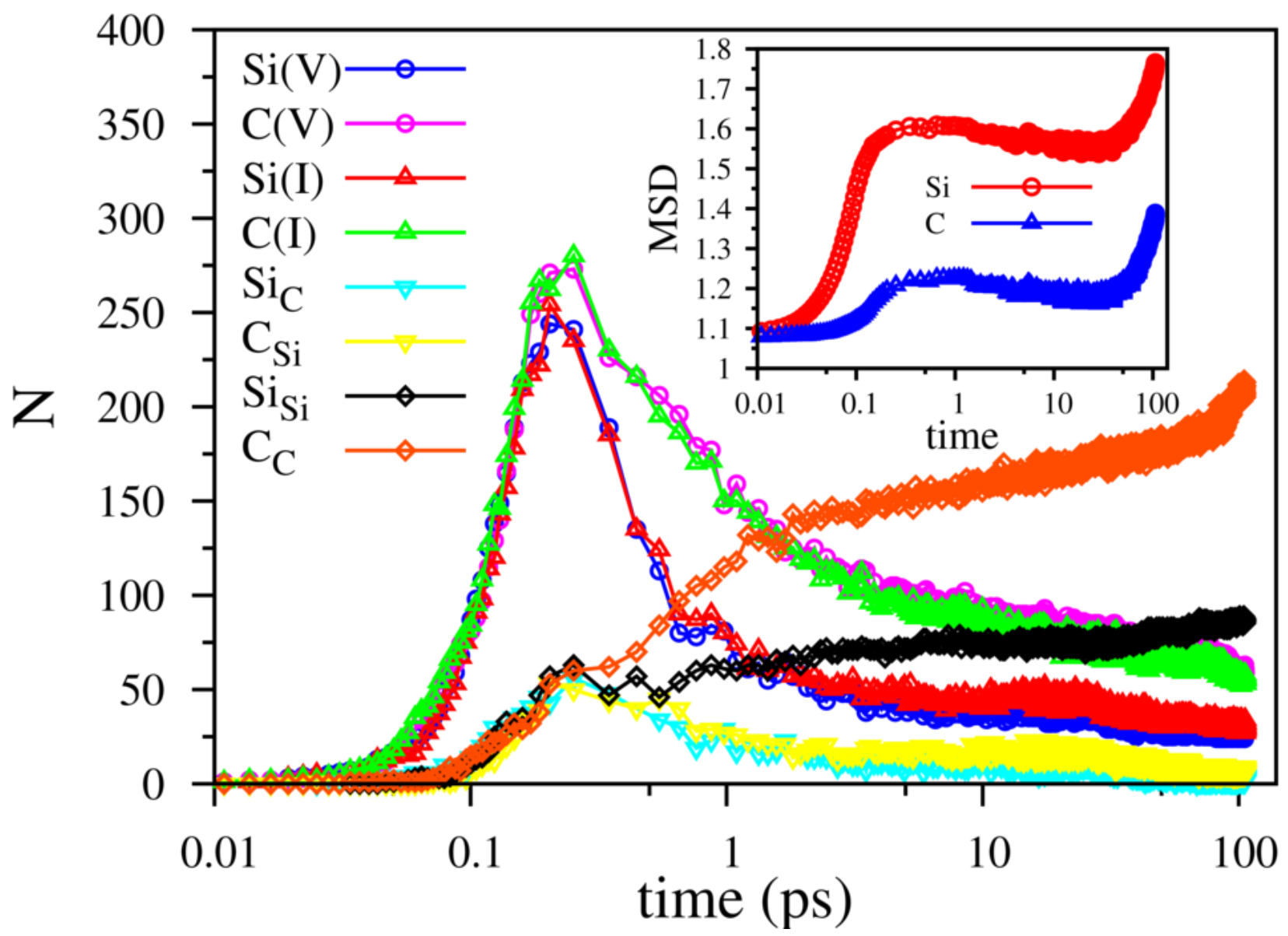

Figure 7. Time dependence of the number of point defects observed in MD displacement cascade simulations at $10 \mathrm{keV}$ PKA at $1200 \mathrm{~K}$ with $\mathrm{GW}$ potential. The inserted figure corresponds to meansquare displacement of $\mathrm{Si}$ (red circles) and $\mathrm{C}$ (blue triangles) atoms as a function of time 
a)

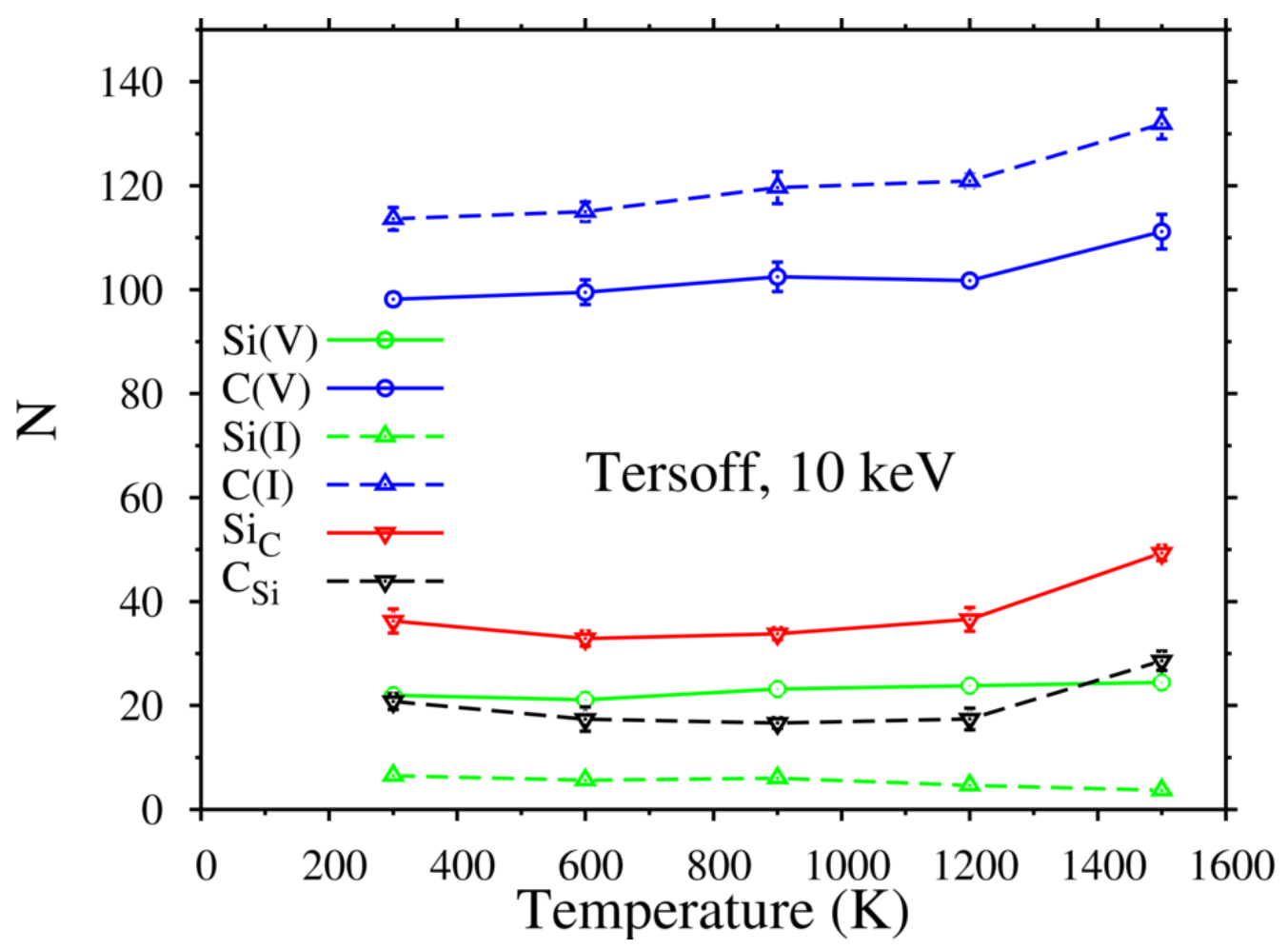

b)

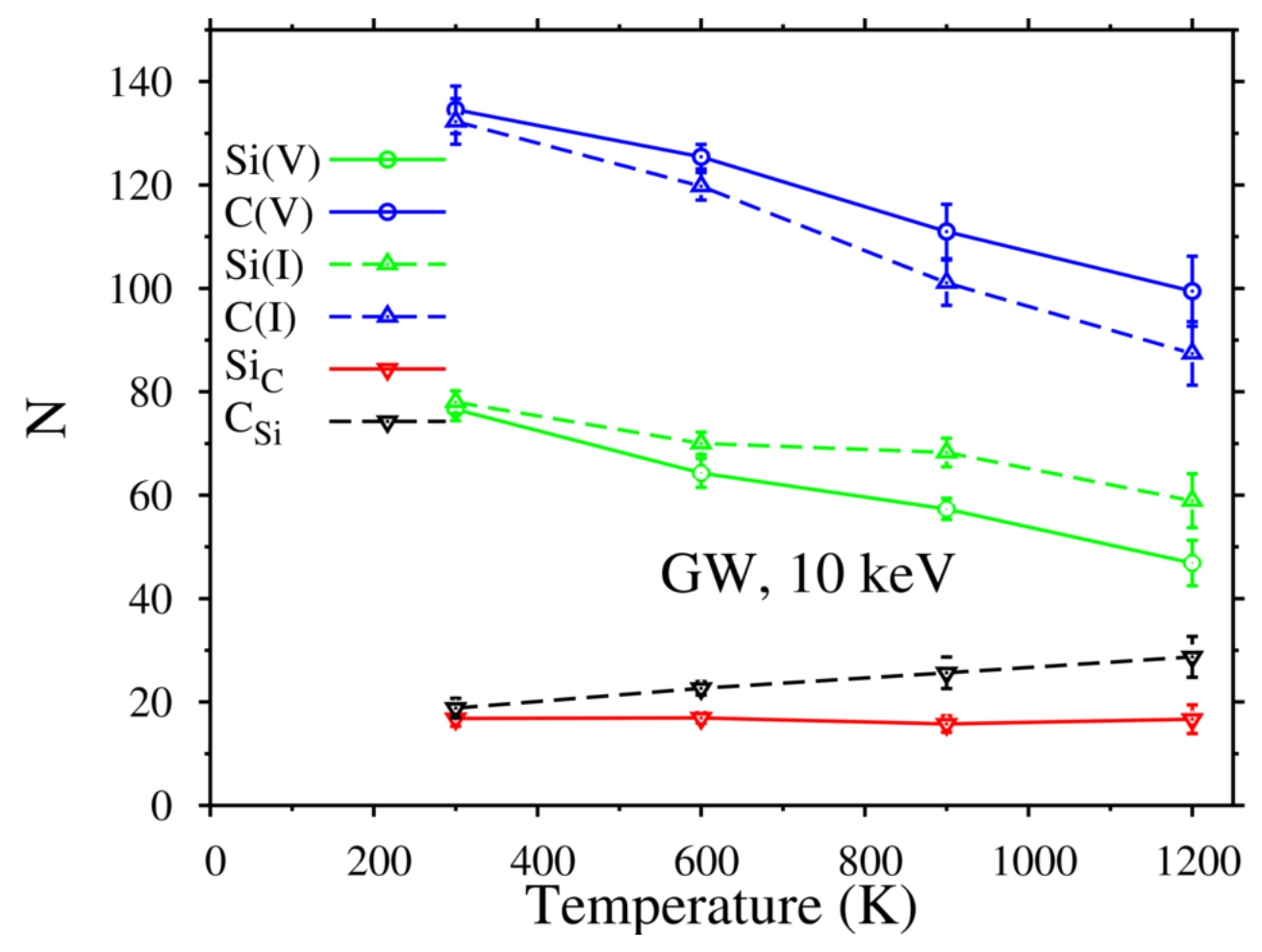

Figure 8 The number of defects at the end of cascade evolution in SiC for 10 pka (a) Tersoff, (b) GW interatomic potentials 
a)

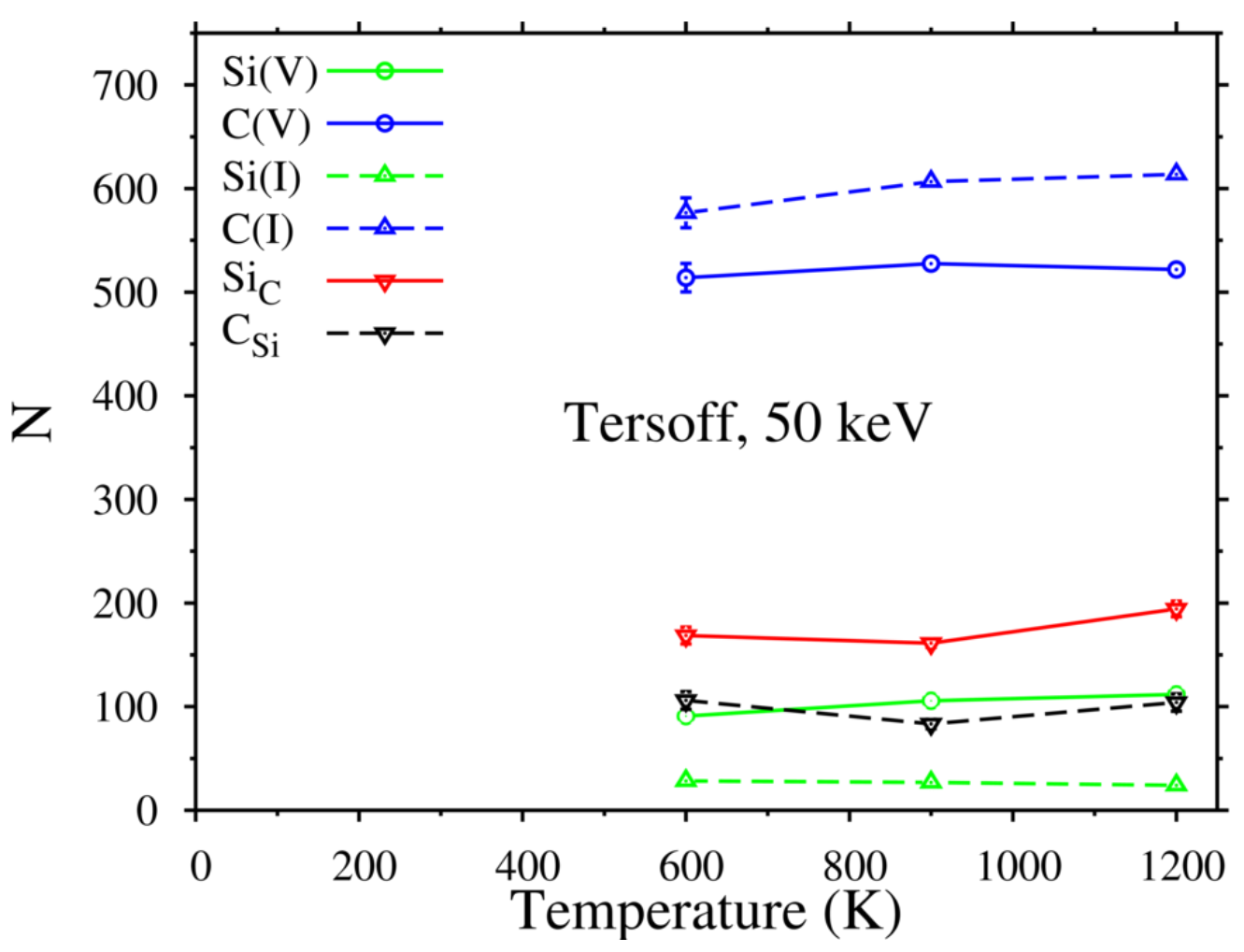

b)

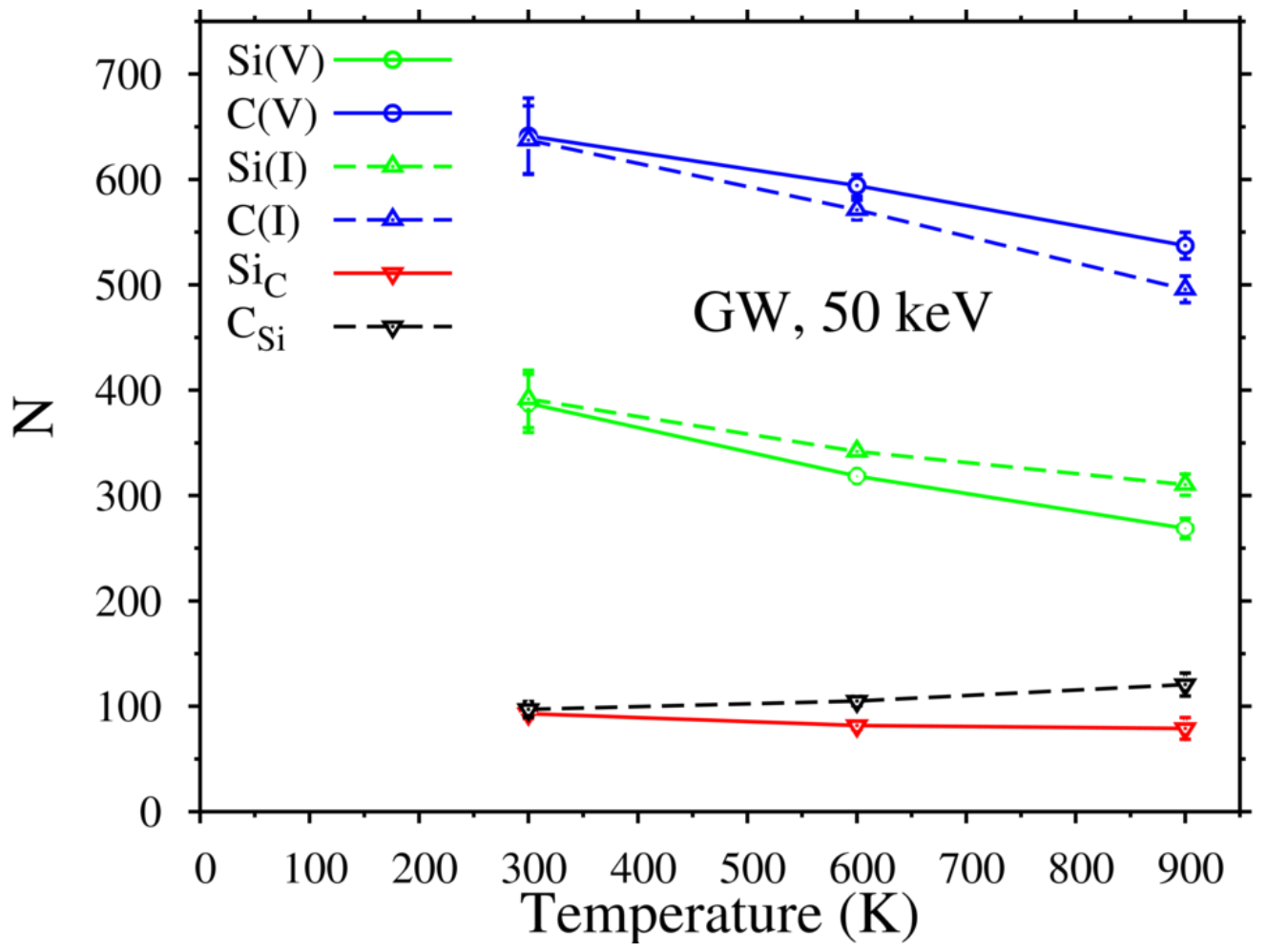

Figure 9 The number of defects at the end of cascade evolution in SiC for 50 pka (a) Tersoff, (b) GW interatomic potentials 